\title{
THE INNER PLETHYSM OF S-FUNCTIONS
}

\author{
D. E. LITTLEWOOD
}

1. Introduction. In a previous paper (2), the inner product of two S-functions $\{\lambda\} \cdot\{\mu\}$ was defined for $(\lambda),(\mu)$ partitions of the same integer $n$. Briefly, the ordinary product $\{\lambda\}\{\mu\}$ of two S-functions corresponds to the analysis of the direct product of two corresponding representations of the full linear group, while the inner product $\{\lambda\} \cdot\{\mu\}$ corresponds to the analysis of the direct product of two representations of the corresponding symmetric group. Thus, if

$$
\chi_{\rho}^{(\lambda)} \chi_{\rho}^{(\mu)}=\sum g_{\lambda \mu \nu} \chi_{\rho}^{(\nu)}
$$

then

$$
\{\lambda\} \cdot\{\mu\}=\sum g_{\lambda \mu \nu}\{\nu\} .
$$

This operation is commutative,

$$
\{\lambda\} \cdot\{\mu\}=\{\mu\} \cdot\{\lambda\},
$$

and distributive with respect to addition,

$$
\{\lambda\} \cdot(\{\mu\}+\{\nu\})=\{\lambda\} \cdot\{\mu\}+\{\lambda\} \cdot\{\nu\} .
$$

Also the symbol $g_{\lambda \mu \nu}$ which arises in this inner product is symmetric with respect to all three.suffixes, so that

$$
g_{\lambda \mu \nu}=g_{\lambda \nu \mu}=g_{\mu \lambda \nu}=g_{\mu \nu \lambda}=g_{\nu \lambda \mu}=g_{\nu \mu \lambda} .
$$

In the case of an inner product of two identical S-functions, $\{\lambda\} .\{\lambda\}$ however, a further analysis is possible. The expression $\{\lambda\} .\{\lambda\}$ corresponds to the direct product of two identical representations of the symmetric group, and such a $\therefore$-rect product can be analysed into its symmetric and skew-symmetric constituents. Thus if $M_{i}$ is the matrix representing a symmetric group element $S_{i}$, then the direct product $M_{i} \times M_{i}$ may be analysed and shown to be equivalent to the direct sum of the second induced matrix and the second compound matrix of $M_{i}$,

$$
M_{i} \times M_{i} \doteq M_{i}^{\{2\}}+M_{i}^{\left\{1^{2}\right\}}
$$

where $\doteq$ denotes equivalence and $\dot{+}$ direct sum.

The expression $\{\lambda\} \cdot\{\lambda\}$ may be analysed correspondingly into two parts ivinch will be denoted respectively by $\{\lambda\} \odot\{2\}$ and $\{\lambda\} \odot\left\{1^{2}\right\}$. The operation which is denoted by the symbol $\odot$ is called inner plethysm, and in the general case is defined as follows.

Received February 8, 1957. 
Let $M_{i}$ be a matrix representation of the symmetric group on $n$ symbols corresponding to the group element $S_{i}$ and let the spur of $M_{i}$ be $\chi^{(\lambda)}\left(S_{i}\right)$. Then the invariant matrix of $M_{i}$ corresponding to the partition $(\mu)$, namely, $M_{i}{ }^{(\mu)}$, is also a matrix representation of the symmetric group, and its spur is a compound character, say

$$
\sum G_{\lambda \mu \nu} \chi^{(\nu)}\left(S_{i}\right)
$$

\section{Definition}

$$
\{\lambda\} \odot\{\mu\}=\sum G_{\lambda \mu \nu}\{\nu\} .
$$

This operation is not commutative. In fact the partitions $(\lambda)$ and $(\nu)$ must be partitions of the same integer $n$, while the partition $(\mu)$ is not so restricted.

The laws governing the combination of this operation with other operations concerning S-functions are in many respects similar to those relating to the ordinary plethysm of S-functions, $\{\lambda\} \otimes\{\mu\}$, except that inner product takes the place of ordinary product.

Ordinary plethysm satisfies the following two laws $(\mathbf{1}, 240)$

$$
\begin{aligned}
& \{\lambda\} \otimes(A+B)=\{\lambda\} \otimes A+\{\lambda\} \otimes B, \\
& \{\lambda\} \otimes(A B)=(\{\lambda\} \otimes A)(\{\lambda\} \otimes B) .
\end{aligned}
$$

If $M$ is an invariant matrix corresponding to $\{\lambda\}$ of a matrix $N$, then the invariant matrix of $M$ corresponding to $\{\mu\}+\{\nu\}$ is by definition equivalent to the direct sum of invariant matrices corresponding to $\{\mu\}$ and $\{\nu\}$ respectively. Expressing the result in terms of S-functions of the latent roots of $N$ this gives

$$
\{\lambda\} \otimes(\{\mu\}+\{\nu\})=\{\lambda\} \otimes\{\mu\}+\{\lambda\} \otimes\{\nu\} .
$$

If now $M$ is taken as a representation of the symmetric group corresponding to the partition $(\lambda)$, the result becomes

$$
\{\lambda\} \odot(\{\mu\}+\{\nu\})=\{\lambda\} \odot\{\mu\}+\{\lambda\} \odot\{\nu\} .
$$

Similarly, considering an invariant matrix of $M$ which is the direct product of invariant matrices corresponding to $\{\mu\},\{\nu\}$ respectively, the result follows, in the first case

$$
\{\lambda\} \otimes(\{\mu\}\{\nu\})=(\{\lambda\} \otimes\{\mu\})(\{\lambda\} \otimes\{\nu\}),
$$

and in the second case, when $M$ is a representation of a symmetric group, the product on the right becoming an inner product,

$$
\{\lambda\} \odot(\{\mu\}\{\nu\})=(\{\lambda\} \odot\{\mu\}) \cdot(\{\lambda\} \odot\{\nu\}) .
$$

Obviously, the simple S-functions $\{\mu\},\{\nu\}$ may be replaced by linear combinations of S-functions, and thus
1.5
$\{\lambda\} \odot(A+B)=\{\lambda\} \odot A+\{\lambda\} \odot B$
1.6
$\{\lambda\} \odot(A B)=(\{\lambda\} \odot A) \cdot(\{\lambda\} \odot B)$. 
The same procedure is available to find the analogue of the equation $(1,290)$

$$
(A+B) \otimes\{\lambda\}=\sum \Gamma_{\mu \nu \lambda}(A \otimes\{\mu\})(B \otimes\{\nu\}) .
$$

With the nomenclature considered above, this equation is obtained by taking a direct sum of two matrices correspondong to $A, B$ respectively. But if these are representations of the symmetric group, the result is

$$
(A+B) \odot\{\lambda\}=\sum \Gamma_{\mu \nu \lambda}(A \odot\{\mu\}) .(B \odot\{\nu\}) .
$$

For a product on the left the known result is

$$
(A B) \otimes\{\lambda\}=\sum g_{\mu \nu \lambda}(A \otimes\{\mu\})(B \otimes\{\nu\})
$$

where

$$
\chi^{(\mu)} \chi^{(\nu)}=\sum g_{\mu \nu \lambda} \chi^{(\lambda)}
$$

which is equivalent to

$$
\{\mu\} \cdot\{\nu\}=\sum g_{\mu \nu \lambda}\{\lambda\} .
$$

To prove this the matrix $M$ is considered as a direct product of two matrices. But if it is the direct product of two representations of the symmetric group, the same reasoning gives

$$
1.8 \quad(A B) \odot\{\lambda\}=\sum g_{\mu \nu \lambda}(A \odot\{\mu\}) .(B \odot\{\nu\}) .
$$

One result is rather remarkable. Ordinary plethysm is associative,

$$
(A \otimes B) \otimes C=A \otimes(B \otimes C) .
$$

If now the basic matrix is taken as a representation of the symmetric group, the two plethysms on the left become inner plethysms. But on the right only the first of the two plethysm signs is changed to inner plethysm. Hence

$$
1.9(A \odot B) \odot C=A \odot(B \otimes C) .
$$

In the place of the associative law the inner plethysm sign is converted to ordinary plethysm.

The evaluation of an expansion of an inner plethysm is not at all easy. A method will be given here, however, for obtaining the expansion of $\{n-1,1\} \odot\{\lambda\}$. For this purpose formulae will be given for evaluating $\{n-1,1\} \odot\{r\}$ and $\{n-1,1\} \odot\left\{1^{r}\right\}$. From either of these results by the aid of inner products the general expansion $\{n-1,1\} \odot\{\lambda\}$ is obtainable.

Some progress may be made by the use of equation 1.9 to evaluate the more general expression $\{\lambda\} \odot\{\mu\}$. However, a much more powerful procedure is obtained by a method of expanding $(\{\lambda\}\{\mu\}) \odot\{\nu\}$.

2. Invariant matrices of permutation matrices. The symmetric group on $n$ symbols has a representation consisting of permutation matrices of degree $n$. The spur of the representation is $\chi^{(n)}+\chi^{(n-1,1)}$, and it is convenient to associate it with the expression

$$
\{n-1\}\{1\}=\{n\}+\{n-1,1\} .
$$


The spurs of the $r$ th induced matrices of these permutation matrices is a sum of symmetric group characters which is associated with the expression

$$
(\{n-1\}\{1\}) \odot\{r\} .
$$

The $r$ th induced matrix is obtained by taking any set of $r$ rows with possible repetitions, and a set of $r$ columns, again with possible repetitions, and taking the permanent of the $r$-rowed square matrix so obtained, allowing a numerical factor $1 / i$ ! for an $i$-fold repeated column. The matrix obtained from such permanents for all choices of $r$ rows and of $r$ columns is the $r$ th induced matrix.

Since each row of a permutation matrix has only one non-zero element, that element being unity, for any choice of $r$ rows there is a unique set of $r$ columns which gives a non-zero permanent, and the permanent in this case is unity. Thus the induced matrix is also a permutation matrix. Further, if the set of $r$ rows has repetitions the set of $r$ columns must have exactly corresponding repetitions.

Let the row of the induced matrix correspond to

$$
\alpha_{1}^{a_{1}} \alpha_{2}^{a_{2}} \ldots \alpha_{i}^{a_{i}}, a_{1}+a_{2}+\ldots+a_{i}=r,
$$

this indicating that the first row is repeated $a_{1}$ times, the second $a_{2}$ times, and so on. This term will be permuted by the symmetric group permutations into every expression which appears in the monomial symmetric function

$$
\sum \alpha_{1}{ }^{a_{1}} \alpha_{2}{ }^{a_{2}} \ldots \alpha_{i}{ }^{a_{i}} \text {. }
$$

The induced matrix can thus be analysed into a direct sum of permutation matrices each corresponding to a monomial symmetric function. These will be considered individually.

Consider first $\sum \alpha_{1}{ }^{r}$. The permutation matrix is simply the matrix of permutation of the $\alpha_{1}$ 's and thus corresponds to

$$
\{n-1\}\{1\} \text {. }
$$

Consider next $\sum \alpha_{1}{ }^{r-1} \alpha_{2}$. The expression $\alpha_{1}{ }^{r-1} \alpha_{2}$ is unaltered by all permutations on the other $n-2$ symbols but is changed by any permutation which involves either $\alpha_{1}$ or $\alpha_{2}$. The corresponding expression is

$$
\{n-2\}\{1\}\{1\} \text {. }
$$

Assuming $r>4, \sum \alpha_{1}{ }^{r-2} \alpha_{2}{ }^{2}$ yields the same result.

Consider next $\sum \alpha_{1}^{r-2} \alpha_{2} \alpha_{3}$. The term $\alpha_{1}{ }^{r-2} \alpha_{2} \alpha_{3}$ is unaltered by any permutation of the other $n-3$ symbols and also by the interchange of $\alpha_{2}$ and $\alpha_{3}$. The corresponding expression is thus

$$
\{n-3\}\{2\}\{1\} \text {. }
$$

The result in the general case may now be inferred. Consider the term

$$
\sum \alpha_{1}^{\lambda_{1}} \alpha_{2}^{\lambda_{2}} \ldots \alpha_{i}^{\lambda_{i}}
$$

where $\left(\lambda_{1}, \lambda_{2}, \ldots, \lambda_{i}\right)$ is any partition of $r$. Let the conjugate partition be $\left(\mu_{1}, \mu_{2}, \ldots, \mu_{j}\right)$, so that $\mu_{1}=i$. 
There are $n-i=n-\mu_{1}$ symbols which do not enter into the expression $\alpha_{1}^{\lambda_{1}} \alpha_{2}^{\lambda_{2}} \ldots \alpha_{i}^{\lambda_{i}}$ and the term is unaltered by the symmetric group of permutations on these symbols. There are $\mu_{1}-\mu_{2}$ indices each equal to $\lambda_{i}$, and again the symmetric group of permutations on these symbols is allowable. There is another set of $\mu_{2}-\mu_{3}$ equal indices, and so on. The corresponding expression is

$$
\left\{n-\mu_{1}\right\}\left\{\mu_{1}-\mu_{2}\right\}\left\{\mu_{2}-\mu_{3}\right\} \ldots\left\{\mu_{j-1}-\mu_{j}\right\}\left\{\mu_{j}\right\} .
$$

Hence

THEOREM I.

$$
(\{n-1\}\{1\}) \odot\{r\}=\sum\left\{n-\mu_{1}\right\}\left\{\mu_{1}-\mu_{2}\right\} \ldots\left\{\mu_{j-1}-\mu_{j}\right\}\left\{\mu_{j}\right\}
$$

summed for all partitions $\left(\mu_{1}, \mu_{2}, \ldots, \mu_{j}\right)$ of $r$.

The following examples illustrate. Since

$$
h_{2}=\sum \alpha_{1}{ }^{2}+\sum \alpha_{1} \alpha_{2}
$$

then

$$
\begin{aligned}
(\{n-1\}\{1\}) \odot\{2\} & =\{n-1\}\{1\}+\{n-2\}\{2\} \\
& =2\{n\}+2\{n-1,1\}+\{n-2,2\} .
\end{aligned}
$$

If $(\lambda)=\left(\lambda_{1}, \lambda_{2}, \ldots, \lambda_{k}\right)$ is a partition of $s$ it is convenient to denote $\left\{n-s, \lambda_{1}, \lambda_{2}, \ldots, \lambda_{k}\right\}$ by $\left[\lambda_{1}, \lambda_{2}, \ldots, \lambda_{k}\right] \equiv[\lambda]$. The above result may then be written

$$
([0]+[1]) \odot\{2\}=2[0]+2[1]+[2] .
$$

Again, corresponding to

$$
\begin{aligned}
h_{3} & =\sum \alpha_{1}{ }^{3}+\sum \alpha_{1}{ }^{2} \alpha_{2}+\sum \alpha_{1} \alpha_{2} \alpha_{3} \\
([0]+[1]) \odot\{3\} & =\{n-1\}\{1\}+\{n-2\}\{1\}\{1\}+\{n-3\}\{3\} \\
& =3[0]+4[1]+2[2]+\left[1^{2}\right]+[3] .
\end{aligned}
$$

Similarly

$$
([0]+[1]) \odot\{4\}=5[0]+7[1]+5[2]+2\left[1^{2}\right]+2[3]+[21]+[4] .
$$

Using equation (7),

$$
([0]+[1]) \odot\{r\}=[1] \odot([r]+[r-1]+\ldots+[1]+[0]) .
$$

Hence

$$
[1] \odot\{r\}=([0]+[1]) \odot(\{r\}-\{r-1\}) .
$$

Thus

$$
\begin{aligned}
& {[1] \odot\{0\}=[0]} \\
& {[1] \odot\{1\}=[1]} \\
& {[1] \odot\{2\}=[0]+[1]+[2]} \\
& {[1] \odot\{3\}=[0]+2[1]+[2]+\left[1^{2}\right]+[3]} \\
& {[1] \odot\{4\}=2[0]+3[1]+3[2]+\left[1^{2}\right]+[3]+[21]+[4]}
\end{aligned}
$$


The compound matrices are in some respects simpler. Consider the $r$ th compound matrix of the permutation matrix which permutes the symbols

$$
\alpha_{1}, \alpha_{2}, \ldots, \alpha_{n} \text {. }
$$

Any set of $r$ distinct rows may be chosen. This gives, for a non-zero determinant, a unique set of $r$ columns. The determinant of the minor is \pm 1 . If it were always +1 the result would be a permutation matrix, but this permutation matrix must be modified to allow for possible factors of the form -1 .

The element in the compound matrix will be unaltered by any permutation of the remaining $n-r$ symbols, but for a permutation of the $r$ symbols there will be a change in sign if the permutation is negative. Thus there is involved the symmetric group on a set of $n-r$ symbols and the negative symmetric group on the set of $r$ symbols. The corresponding expression is

$$
(\{n-1\}\{1\}) \odot\left\{1^{r}\right\}=\{n-r\}\left\{1^{r}\right\}=\left\{n-r+1,1^{r-1}\right\}+\left\{n-r, 1^{r}\right\} .
$$

Again from equation ( 7$)$,

$$
([0]+[1]) \odot\left\{1^{r}\right\}=[1] \odot\left(\left\{1^{r}\right\}+\left\{1^{r-1}\right\}\right) .
$$

Hence

THEOREM II.

$$
\{n-1,1\} \odot\left\{1^{r}\right\}=\left\{n-r, 1^{r}\right\} .
$$

To illustrate, the expression for $[1] \odot\{3\}$ will be obtained by the use of Theorem II.

Since

$$
\{3\}=\{1\}^{3}+\left\{1^{3}\right\}-2\{1\}\left\{1^{2}\right\}
$$

therefore

$$
\begin{aligned}
{[1] \odot\{3\} } & =[1] \odot\left(\{1\}^{3}+\left\{1^{3}\right\}-2\{1\}\left\{1^{2}\right\}\right) \\
& =[1] .[1] \cdot[1]+\left[1^{3}\right]-2[1] \cdot\left[1^{2}\right] .
\end{aligned}
$$

Since

$$
[1] \cdot[1]=[0]+[1]+[2]+\left[1^{2}\right]
$$

therefore

$$
\begin{aligned}
{[1] \odot\{3\} } & =\left[1^{3}\right]+[1] \cdot\left([0]+[1]+[2]-\left[1^{2}\right]\right) \\
& =\left[1^{3}\right]+[1]+[0]+[2]+\left[1^{2}\right]+[3]-\left[1^{3}\right] \\
& =[0]+2[1]+[2]+\left[1^{2}\right]+[3],
\end{aligned}
$$

which conforms with the result obtained by the use of Theorem I.

By evaluating inner products in this way the general expansion $\{n-1,1\} \odot\{\lambda\}$ may be evaluated either from Theorem I or from Theorem II.

A generalization of the method to evaluate $\{\lambda\} \odot\{\mu\}$ in the general case is not apparent. However, some progress may be made by the use of equation 1.9 . 
Thus, since

$$
[1] \odot\left\{1^{2}\right\}=\left[1^{2}\right]
$$

therefore

$$
\begin{aligned}
{\left[1^{2}\right] \odot\left\{1^{2}\right\} } & =\left([1] \odot\left\{1^{2}\right\}\right) \odot\left\{1^{2}\right\}=[1] \odot\left(\left\{1^{2}\right\} \otimes\left\{1^{2}\right\}\right) \\
& =[1] \odot\left\{21^{2}\right\}=[1] \odot\left(\{1\}\left\{1^{3}\right\}-\left\{1^{4}\right\}\right) \\
& =[1] \cdot\left[1^{3}\right]-\left[1^{4}\right] \\
& =\left[1^{2}\right]+[21]+\left[1^{3}\right]+\left[21^{2}\right] .
\end{aligned}
$$

Evaluation of $\left[1^{2}\right] \odot\{\lambda\}$ may be performed by this method. The evaluation of $[2] \odot\{\lambda\}$ is a little more complicated.

Thus, since

$$
[1] \odot\{2\}=[0]+[1]+[2]
$$

therefore

$$
\begin{aligned}
([0]+[1]+[2]) \odot\left\{1^{2}\right\} & =[1] \odot\left(\{2\} \otimes\left\{1^{2}\right\}\right) \\
& =[1] \odot\{31\},
\end{aligned}
$$

which may be evaluated as described above to give

$$
2[1]+2[2]+3\left[1^{2}\right]+[3]+2[21]+\left[1^{3}\right]+[31] .
$$

But also

$$
\begin{aligned}
([0]+[1]+[2]) \odot\left\{1^{2}\right\} & =([0]+[1]) \odot\left\{1^{2}\right\}+([0]+[1]) \cdot[2]+[2] \odot\left\{1^{2}\right\} \\
& =2[1]+2[2]+2\left[1^{2}\right]+[3]+[21]+[2] \odot\left\{1^{2}\right\} .
\end{aligned}
$$

Hence

$$
[2] \odot\left\{1^{2}\right\}=\left[1^{2}\right]+[21]+\left[1^{3}\right]+[31] .
$$

Theoretically such procedures will allow the evaluation of $\{\lambda\} \odot\{\mu\}$ in every case, but in practice the calculation may become extremely involved.

The general method, however, is applicable to any permutation representation, although the only one so far considered has been the permutation of the actual symbols, which corresponds to the expression $\{n-1\}\{1\}$.

Consider the subgroup corresponding to $\{n-r\}\{r\}$. The corresponding permutation representation permutes the sets of $r$ symbols such as $\alpha_{1}, \alpha_{2}, \ldots, \alpha_{r}$. The second induced matrix of the representation will correspond to the expression

$$
(\{n-r\}\{r\}) \odot\{2\} .
$$

Taking a pair of rows from the permutation matrix, these will correspond to two sets of $r$ symbols $\alpha_{1}, \alpha_{2}, \ldots, \alpha_{r}$ and $\alpha_{1}{ }^{\prime}, \alpha_{2}{ }^{\prime}, \ldots, \alpha_{r}{ }^{\prime}$. If there are $r-i$ symbols common to the two sets, then also for the corresponding columns which give a non-zero element the two sets of $r$ symbols will have exactly $r-i$ symbols in common. The second induced matrix is therefore reducible, the constituent matrices corresponding to the different values of $i$. It is therefore pertinent to consider these individually. 
Consider the two sets of symbols

$$
\alpha_{1}, \alpha_{2}, \ldots, \alpha_{i}, \alpha_{i+1}, \ldots, \alpha_{r} ; \alpha_{1}^{\prime}, \alpha_{2}^{\prime}, \ldots, \alpha_{i}^{\prime}, \alpha_{i+1}, \ldots, \alpha_{r} .
$$

the pair of sets is invariant for any permutation confined to the set $\alpha_{1}, \ldots, \alpha_{i}$, or to the set $\alpha_{1}{ }^{\prime}, \ldots, \alpha_{i}{ }^{\prime}$, or to the set $\alpha_{i+1}, \ldots, \alpha_{r}$. It is also invariant for the interchange of the first two sets of $i$ symbols. The corresponding expression is therefore

$$
(\{i\} \otimes\{2\})\{r-i\}\{n-r-i\} .
$$

Hence

THEOREM III.

$$
(\{n-r\}\{r\}) \odot\{2\}=\sum_{i=0}^{r}(\{i\} \otimes\{2\})\{r-i\}\{n-r-i\} .
$$

The second compound matrix gives similarly

\section{THEOREM IV.}

$$
(\{n-r\}\{r\}) \odot\left\{1^{2}\right\}=\sum_{i=1}^{r}\left(\{i\} \otimes\left\{1^{2}\right\}\right)\{r-i\}\{n-r-i\} .
$$

The case $i=0$ can be omitted since $\{0\} \otimes\left\{1^{2}\right\}=0$.

3. Extension to products of S-functions. These theorems can be generalized. There is a matrix representation of this subgroup which is the direct product of a representation of the symmetric group on the first $r$ symbols corresponding to the partition $(\lambda)$ of $r$, and a representation of the symmetric group on the other $n-r$ symbols corresponding to the partition $(\mu)$ of $n-r$. The corresponding representation of the symmetric group on $n$ symbols is constructed by taking the direct sum of $n ! / r !(n-r)$ ! such representations corresponding to the conjugate subgroups and allowing for the permutation of these representations as well as the matrix products in the various representations. The representation ${ }^{1}$ so obtained, of degree $f^{(\lambda)} f^{(\mu)} n ! / r !(n-r)$ ! corresponds to the expression

$$
\{\lambda\}\{\mu\} .
$$

The second induced matrix of this representation corresponds to

$$
(\{\lambda\}\{\mu\}) \odot\{2\} .
$$

It is convenient to consider at the same time the second compound matrix, corresponding to

$$
(\{\lambda\}\{\mu\}) \odot\left\{1^{2}\right\}
$$

and the following Theorem is obtained.

'See (6) where this representation is extensively used. 
Theorem V. If $(\nu)$ is a partition of 2 ,

$$
\begin{aligned}
& (\{\lambda\}\{\mu\}) \odot\{\nu\}=\sum g_{\xi \eta \eta \nu \nu} \Gamma_{\alpha \theta \lambda} \Gamma_{\beta \phi \mu}(\{\alpha\} \odot\{\xi\})(\{\beta\} \odot\{\eta\})[(\{\theta\} \cdot\{\phi\}) \otimes\{\zeta\}] \\
& \quad+\sum \frac{1}{2}\left(\Gamma_{\alpha \theta \lambda}^{2} \Gamma_{\beta \phi \mu}^{2}-\Gamma_{\alpha \theta \lambda} \Gamma_{\beta \phi \mu}\right)(\{\alpha\} \cdot\{\alpha\})(\{\beta\} \cdot\{\beta\})(\{\phi\} \cdot\{\theta\})(\{\phi\} \cdot\{\theta\}) \\
& +\sum \Gamma_{\alpha \theta \lambda} \Gamma_{\alpha^{\prime} \theta^{\prime} \lambda} \Gamma_{\beta \phi \mu} \Gamma_{\beta^{\prime} \phi^{\prime} \mu}\left(\{\alpha\} \cdot\left\{\alpha^{\prime}\right\}\right)\left(\{\beta\} \cdot\left\{\beta^{\prime}\right\}\right)(\{\phi\} \cdot\{\theta\})\left(\left\{\phi^{\prime}\right\} .\left\{\theta^{\prime}\right\}\right) .
\end{aligned}
$$

In this enunciation $\Gamma_{\alpha \beta \lambda}$ is the coefficient of $\{\lambda\}$ in $\{\alpha\}\{\theta\}, g_{\xi \eta\}_{\nu}}$ is the coefficient of $\{\nu\}$ in $\{\xi\} \cdot\{\eta\} \cdot\{\zeta\}$ so that $(\xi),(\eta),(\zeta)$ must be partitions of 2 . Instead of taking $(\lambda)$ and $(\mu)$ as partitions of $r$ and $n-r$ it is rather simpler to take $(\lambda)$ as a partition of $m$ and $(\mu)$ as a partition of $n$. If $(\theta)$ is a partition of $i$, then so is $(\phi)$, and also $\left(\theta^{\prime}\right)$ and $\left(\phi^{\prime}\right)$ in the last summation. Then $(\alpha)$, $\left(\alpha^{\prime}\right)$ are partitions of $m-i$, and $(\beta),\left(\beta^{\prime}\right)$ partitions of $n-i$. In the last two summations, if $(\alpha)$ and $\left(\alpha^{\prime}\right),(\beta)$ and $\left(\beta^{\prime}\right),(\theta)$ and $\left(\theta^{\prime}\right),(\phi)$ and $\left(\phi^{\prime}\right)$ are interchanged an identical term is obtained, but only one of the two identical terms is included in the summation. The cases for which $(\alpha)=\left(\alpha^{\prime}\right),(\beta)=\left(\beta^{\prime}\right)$, $(\theta)=\left(\theta^{\prime}\right),(\phi)=\left(\phi^{\prime}\right)$ are excluded from the last summation.

The value of $(\{\lambda\}\{\mu\}) \odot\{2\}$ or $(\{\lambda\}\{\mu\}) \odot\left\{1^{2}\right\}$ is obtained by summing the permanents or determinants of the two rowed principal minors of the representation corresponding to $\{\lambda\}\{\mu\}$. Consider any pair of rows. Corresponding to $(\lambda)$ there are, associated with these two rows, two sets of $m$ symbols. Suppose that $m-i$ symbols are common to the two sets. For non-zero results permutations must be confined to those which permute these $m-i$ symbols, permute the $n-i$ symbols which occur in neither set, permute each set of $i$ symbols which occur in one set only, or interchange these two sets of $i$ symbols.

Proceeding from the symmetric group on $m$ symbols to the subgroup which permutes separately the $m-i$ and the $i$ symbols, the representation corresponding to $\{\lambda\}$ reduces to various representations corresponding to $\{\alpha\}\{\theta\}$ where $(\alpha)$ is a partition of $m-i$ and $(\theta)$ is a partition of $i$. Such a representation occurs with frequency $\Gamma_{\alpha \theta \lambda}$. We are thus led to a term $\sum \Gamma_{\alpha \theta \lambda}\{\alpha\}\{\theta\}$. Similarly for the second row there is a term $\sum \Gamma_{\alpha^{\prime} \theta^{\prime} \lambda}\left\{\alpha^{\prime}\right\}\left\{\theta^{\prime}\right\}$.

To a permutation among the $m-i$ symbols there corresponds the direct product of the representations corresponding to each of the two rows. This leads to

$$
\sum \Gamma_{\alpha \theta \lambda} \Gamma_{\alpha^{\prime} \theta^{\prime} \lambda}\left(\{\alpha\} \cdot\left\{\alpha^{\prime}\right\}\right)\{\theta\}\{\phi\} .
$$

Combining this with an equivalent result in relation to the partition $(\mu)$ and remembering that for a set of $i$ symbols, for one row the representation corresponds to $(\theta)$, being obtained from the representation corresponding to $(\lambda)$, while for the other row the representation corresponds to some $(\phi)$, obtained from the representation corresponding to $(\mu)$, it is clear that the final result is that given in the last summation in the enunciation of Theorem $\mathrm{V}$.

But allowance must be made for the possible interchange of the two sets of $i$ symbols. This interchange is only possible when $(\alpha)=\left(\alpha^{\prime}\right),(\beta)=\left(\beta^{\prime}\right),(\theta)=\left(\theta^{\prime}\right)$, $(\phi)=\left(\phi^{\prime}\right)$. In this case $\{\alpha\} \cdot\{\alpha\}$ is replaced by $\{\alpha\} \odot\{2\}+\{\alpha\} \odot\left\{1^{2}\right\}$, of which 
the first involves a plus, the second a minus sign for the interchange. If $\Gamma_{\alpha \theta \lambda}$ is 0 or 1 in every case, this is sufficient.

Similarly $\{\beta\} .\{\beta\}$ is replaced by $\{\beta\} \odot\{2\}+\{\beta\} \odot\left\{1^{2}\right\}$. But the ordinary product $(\{\phi\} .\{\theta\})(\{\phi\} .\{\theta\})$ is replaced by $\left(\{\phi\} .\{\theta\} \otimes\{2\}+(\{\phi\} .\{\theta\}) \odot\left\{1^{2}\right\}\right.$.

Of the three alternative signs, for $(\{\lambda\}\{\mu\}) \odot\{2\}$ either all must be positive or exactly two negative, and for $(\{\lambda\}\{\mu\}) \odot\left\{1^{2}\right\}$ either one or three must be negative. This accounts for the coefficient $\gamma_{\xi \eta \xi \nu}$.

Special consideration must be given to the case when $\Gamma_{\alpha \theta \lambda} \Gamma_{\beta \phi \mu}>1$. If, for example, $\Gamma_{\alpha \theta \lambda}>1$ then terms corresponding to $\{\alpha\}\{\theta\}$ occur more than once. In the detailed analysis these will correspond to different Young Tableaux. But the interchange of $(\alpha)$ with $\left(\alpha^{\prime}\right)$ etc., is only allowable if the same Young Tableau is concerned in each case. The reduction from products to plethysms can only occur in $\Gamma_{\alpha \theta \lambda} \Gamma_{\beta \phi \mu}$ cases. There remain $\Gamma_{\alpha \theta \lambda}^{2} \Gamma_{\beta \phi \mu}^{2}-\Gamma_{\alpha \theta \lambda} \Gamma_{\beta \phi \mu}$ cases where in the two rows there is a difference in the corresponding Young Tableaux. These cases remain as ordinary or inner products, but with only half the frequency. This completes the proof of the Theorem.

As an example consider $\left(\left\{31^{2}\right\}\{1\}\right) \odot\{2\}$. For $\{\alpha\}=\left\{31^{2}\right\}$ the terms are

$$
\left(\left\{31^{2}\right\} \odot\{2\}\right)(\{1\} \odot\{2\})+\left(\left\{31^{2}\right\} \odot\left\{1^{2}\right\}\right)\left(\{1\} \odot\left\{1^{2}\right\}\right) .
$$

The second term is zero. To evaluate the first, note that

$$
\begin{aligned}
\left\{31^{2}\right\} \odot\{2\} & =\left(\{41\} \odot\left\{1^{2}\right\}\right) \odot\{2\}=\{41\} \odot\left(\left\{1^{2}\right\} \otimes\{2\}\right) \\
& =\{41\} \odot\left(\left\{2^{2}\right\}+\left\{1^{4}\right\}\right) \\
& =\left\{31^{2}\right\} .\left\{31^{2}\right\}-\{41\} .\left\{21^{3}\right\}+\left\{1^{5}\right\} \\
& =\{5\}+\{41\}+2\{32\}+\left\{2^{2} 1\right\}+\left\{1^{5}\right\} .
\end{aligned}
$$

The term is thus

$$
\begin{aligned}
& \{6\}+2\{51\}+3\{42\}+\left\{41^{2}\right\}+2\left\{3^{2}\right\}+3\{321\}+\left\{2^{3}\right\}+\left\{2^{2} 1^{2}\right\}+\left\{21^{4}\right\}+\left\{1^{6}\right\} . \\
& \text { For }\{\alpha\}=\left\{\alpha^{\prime}\right\}=\{31\} \text { the terms are } \\
& \begin{aligned}
(\{31\} \odot\{2\})\{2\}+\left(\{31\} \odot\left\{1^{2}\right\}\right)\left\{1^{2}\right\} \\
=\left(\{4\}+\{31\}+\left\{2^{2}\right\}\right)\{2\}+\left\{21^{2}\right\}\left\{1^{2}\right\} \\
=\{6\}+2\{51\}+3\{42\}+\left\{41^{2}\right\}+\left\{3^{2}\right\} \\
+3\{321\}+2\left\{2^{3}\right\}+\left\{31^{3}\right\}+\left\{2^{2} 1^{2}\right\}+\left\{21^{4}\right\} .
\end{aligned}
\end{aligned}
$$

The case $\{\alpha\}=\left\{\alpha^{\prime}\right\}=\left\{21^{2}\right\}$ gives

$$
\left(\left\{21^{2}\right\} \odot\{2\}\right)\{2\}+\left(\left\{21^{2}\right\} \odot\left\{1^{2}\right\}\right)\left\{1^{2}\right\}
$$

which yields an identical result.

The only other case is $\{\alpha\}=\{31\},\left\{\alpha^{\prime}\right\}=\left\{21^{2}\right\}$. The rows are not now interchangeable and the terms are

$$
\begin{aligned}
\left(\{31\} \cdot\left\{21^{2}\right\}\right)\{1\}\{1\}= & \left(\{31\}+\left\{2^{2}\right\}+\left\{21^{2}\right\}+\left\{1^{4}\right\}\right)\{1\}\{1\} \\
= & \{51\}+3\{42\}+3\left\{41^{2}\right\}+2\left\{3^{2}\right\}+6\{321\}+2\left\{2^{3}\right\} \\
& +4\left\{31^{3}\right\}+4\left\{2^{2} 1^{2}\right\}+3\left\{21^{4}\right\}+\left\{1^{6}\right\} .
\end{aligned}
$$


Hence

$$
\begin{aligned}
\left(\left\{31^{2}\right\}\{1\}\right) \odot\{2\}=3\{6\}+ & 7\{51\}+12\{42\}+6\left\{41^{2}\right\}+6\left\{3^{2}\right\}+15\{321\} \\
& +7\left\{2^{3}\right\}+6\left\{31^{3}\right\}+7\left\{2^{2} 1^{2}\right\}+6\left\{21^{4}\right\}+2\left\{1^{6}\right\} .
\end{aligned}
$$

If this is expressed as

$$
\begin{aligned}
\left(\left\{31^{2}\right\}\{1\}\right) \odot\{2\}= & \left(\left\{41^{2}\right\}+\left\{31^{3}\right\}+\{321\}\right) \odot\{2\} \\
= & \left\{41^{2}\right\} \odot\{2\}+\left\{31^{3}\right\} \odot\{2\}+\left\{41^{2}\right\} .\left\{31^{3}\right\}+ \\
& \left\{41^{2}\right\} .\{321\}+\left\{31^{3}\right\} .\{321\}+\{321\} \odot\{2\},
\end{aligned}
$$

the evaluation of the first five terms and substitution leads to

$$
\begin{aligned}
\odot\{2\}=\{6\}+2\{51\}+ & 3\{42\}+\left\{41^{2}\right\}+\left\{3^{2}\right\}+3\{321\} \\
+ & 2\left\{2^{3}\right\}+\left\{31^{3}\right\}+\left\{2^{2} 1^{2}\right\}+\left\{21^{4}\right\}+\left\{1^{6}\right\} .
\end{aligned}
$$

4. Plethysm and inner plethysm. In the case when $(\lambda)=(\mu)$, the left hand side of the equation of Theorem $\mathrm{V}$ becomes

$$
\begin{aligned}
(\{\lambda\}\{\lambda\}) \odot\{\nu\}= & \left(\{\lambda\} \otimes\{2\}+\{\lambda\} \otimes\left\{1^{2}\right\}\right) \odot\{\nu\} \\
= & (\{\lambda\} \otimes\{2\}) \odot\{\nu\}+\left(\{\lambda\} \otimes\left\{1^{2}\right\}\right) \odot\{\nu\}+ \\
& (\{\lambda\} \otimes\{2\}) .\left(\{\lambda\} \otimes\left\{1^{2}\right\}\right) .
\end{aligned}
$$

By a careful analysis of the terms which appear on the right of the equation it is possible to separate these so as to give expansions for the expressions $(\{\lambda\} \otimes\{\mu\}) \odot\{\nu\}$ when $(\mu),(\nu)$ are partitions of 2 .

It is more convenient to start with the expansion

$$
\begin{array}{r}
4.1[\{\lambda\}\{\lambda\}] .[\{\lambda\}\{\lambda\}]=\sum \Gamma_{\alpha \theta \lambda} \Gamma_{\alpha^{\prime} \theta^{\prime} \lambda} \Gamma_{\beta \phi \mu} \Gamma_{\beta^{\prime} \phi^{\prime} \mu}\left(\{\alpha\} .\left\{\alpha^{\prime}\right\}\right)\left(\{\beta\} .\left\{\beta^{\prime}\right\}\right) \\
(\{\phi\} .\{\theta\})\left(\left\{\phi^{\prime}\right\} .\left\{\theta^{\prime}\right\}\right) .
\end{array}
$$

Consider the set of 8 symbols

$$
\alpha, \theta, \phi, \beta, \beta^{\prime}, \phi^{\prime}, \theta^{\prime}, \alpha^{\prime} .
$$

Let $T$ be an operation which reverses the order of these 8 symbols, and $S$ an operation which permutes them cyclically, moving them two steps at a time, so that, for example, $\alpha \rightarrow \phi \rightarrow \beta^{\prime}$ etc. Then

$$
T^{2}=I=S^{4}, \quad S T=T S^{3} .
$$

The operations $S, T$ generate a group $G$ of order 8 , and each term on the right of 4.1 is converted into an equal term by the operations of this group.

In general the terms on the right of 4.1 are equal in sets of 8 according to the operations of this group. But it may happen that, by reason of certain equalities among the 8 symbols, certain operations of $G$ convert the term into the same term. If the operations which leave the term invariant form a subgroup $\Gamma$ of $G$ of order $\gamma$, the term is repeated only $8 / \gamma$ times. When the term is repeated 8 times just one of the 8 terms is included in $(\{\lambda\} \otimes\{\mu\}) \odot\{\nu\}$ for any partitions $(\mu),(\nu)$ of 2 . But when $\gamma>1$, the term must be further re- 
duced by converting products into plethysms or inner products into inner plethysms.

The result will be enunciated as a Theorem. The full result is rather complicated owing to the need to provide for all the exceptional cases that can arise, in order to give complete generality. It is thought worth while to give this result in its full generality since it is a basic result concerning the interaction of operations with S-functions. To save space, however, proofs will be omitted. Because of the multiplicity of special cases such proof becomes intricate and involved. The principle involved is straightforward, representing only the operation of the appropriate symmetrizing operator on the general term.

THeOREM VI. If $(\lambda),(\mu)$ are partitions of 2 , the expansion of $(\{\lambda\} \otimes\{\mu\}) \odot\{\nu\}$ is obtained from the right hand side of equation 4.1 by selecting one from each set of $8 / \gamma$ equal terms which appear, and operating on this term with the appropriate symmetrizing operator.

The appropriate symmetrizing operators will now be described, and the effects on the term listed for all possible subgroups.

For simplicity, suppose first that $\Gamma_{\alpha \theta \lambda}$ and all similar coefficients are either 0 or 1 .

(1) If $\Gamma=I$, the term is taken unchanged.

(2) If $\Gamma=I, T$ the 8 symbols may be taken as

$$
\alpha, \theta, \phi, \beta, \beta, \phi, \theta, \alpha \text {. }
$$

The term is replaced by

$$
\sum(\{\alpha\} \odot\{\xi\})(\{\beta\} \odot\{\eta\})[(\{\theta\} .\{\phi\}) \otimes(\{\xi\} .\{\eta\} .\{\nu\})],
$$

summed for all partitions $(\xi),(\eta)$ of 2 .

(3) If $\Gamma=I, S^{2}$ the 8 symbols can be taken as

$$
\alpha, \theta, \phi, \beta, \alpha, \theta, \phi, \beta \text {. }
$$

The term is replaced by

$$
\sum[(\{\alpha\} .\{\beta\}) \otimes\{\xi\}][(\{\theta\} .\{\phi\}) \otimes\{\xi\}] .
$$

The summation in this and other cases is for $(\xi)=(2),\left(1^{2}\right)$ and when it appears, for $(\eta)=(2),\left(1^{2}\right)$.

(4) If $\Gamma=I, T S$ the symbols can be expressed as

$$
\alpha, \theta, \phi, \phi, \theta, \alpha, \alpha^{\prime}, \alpha^{\prime} \text {. }
$$

The term is replaced by

$$
\sum\left[\left(\{\alpha\} .\left\{\alpha^{\prime}\right\}\right) \otimes\{\xi\}\right][(\{\theta\} .\{\phi\}) \otimes(\{\xi\} \cdot\{\mu\})] .
$$

(5) If $\Gamma=I, S, S^{2}, S^{3}$ the symbols can be expressed as

$$
\alpha, \theta, \alpha, \theta, \alpha, \theta, \alpha, \theta \text {. }
$$


The term is replaced by

$$
\sum[(\{\alpha\} \cdot\{\theta\}) \otimes\{\xi\}] \otimes(\{\mu\} \cdot\{\nu\}) .
$$

(6) If $\Gamma=I, S^{2}, T, T S^{2}$ the symbols can be expressed as

$$
\alpha, \theta, \theta, \alpha, \alpha, \theta, \theta, \alpha \text {. }
$$

The term is replaced by

$$
\begin{aligned}
4.7 \quad\left[(\{\alpha\} \odot\{2\}) \otimes\{\nu\}+\left(\{\alpha\} \odot\left\{1^{2}\right\}\right) \otimes\{\nu\}\right][(\{\theta\} \odot\{2\}) \otimes\{\nu\} \\
\left.+\left(\{\theta\} \odot\left\{1^{2}\right\}\right) \otimes\{\nu\}\right] \\
+\left[(\{\alpha\} \odot\{2\}) \otimes\left(\{\nu\} .\left\{1^{2}\right\}\right)\right. \\
\left.+\left(\{\alpha\} \odot\left\{1^{2}\right\}\right) \otimes\left(\{\nu\} .\left\{1^{2}\right\}\right)\right](\{\theta\} \odot\{2\})\left(\{\theta\} \odot\left\{1^{2}\right\}\right) \\
+\left[(\{\theta\} \odot\{2\}) \otimes\left(\{\nu\} .\left\{1^{2}\right\}\right)\right. \\
+\left(\{\theta\} \odot\left\{1^{2}\right\}\right) \otimes\left(\{\nu\} .\left\{1^{2}\right\}\right](\{\alpha\} \odot\{2\})\left(\{\alpha\} \odot\left\{1^{2}\right\}\right) \\
+(\{\alpha\} \odot\{2\})\left(\{\alpha\} \odot\left\{1^{2}\right\}\right)(\{\theta\} \odot\{2\})\left(\{\theta\} \odot\left\{1^{2}\right\}\right) .
\end{aligned}
$$

(7) If $\Gamma=I, S^{2}, T S, S T$ the symbols can be expressed as

$$
\alpha, \alpha, \beta, \beta, \alpha, \alpha, \beta, \beta \text {. }
$$

The term is replaced by

$$
\sum(\{\alpha\} \cdot\{\beta\}) \otimes\{\xi\} \otimes\{\mu\} .
$$

(8) Lastly when $\Gamma=G$ so that all 8 symbols represent the same partition $(\alpha)$, the term is replaced by

$$
\begin{gathered}
4.9(\{\alpha\} \odot\{2\}) \otimes\{\mu\} \otimes\{\nu\}+\left(\{\alpha\} \odot\left\{1^{2}\right\}\right) \otimes\{\mu\} \otimes\{\nu\}+\left[\left(\{\alpha\} \odot\left\{1^{2}\right\}\right)\right. \\
\otimes\{\mu\}][(\{\alpha\} \odot\{2\}) \otimes\{\mu\}]+\left[(\{\alpha\} \odot\{2\})\left(\{\alpha\} \odot\left\{1^{2}\right\}\right)\right] \otimes\{\nu\}+ \\
\\
{\left[(\{\alpha\} \odot\{2\}) \otimes\{\tilde{\mu}\}+\left(\{\alpha\} \odot\left\{1^{2}\right\}\right) \otimes\{\tilde{\mu}\}\right](\{\alpha\} \odot\{2\})\left(\{\alpha\} \odot\left\{1^{2}\right\}\right) .}
\end{gathered}
$$

where $\{\tilde{\mu}\}=\{\mu\} \cdot\left\{1^{2}\right\}$.

The cases when $\Gamma_{\alpha \theta \lambda}$ or a similar coefficient is $>1$ must be considered for the sake of completeness, although it seems really to be of academic importance only. The simplest case occurs with

$$
(\{321\} \otimes\{2\}) \odot\{2\}
$$

which involves representations of the symmetric group on 12 symbols. In the application of the theorem several hundreds of different cases arise.

The required changes occur for subgroups $\Gamma$ when the coefficient

$$
\Gamma_{\alpha \theta \lambda} \Gamma_{\alpha^{\prime} \theta^{\prime} \lambda} \Gamma_{\beta \phi \lambda} \Gamma_{\beta^{\prime} \phi^{\prime} \lambda}
$$

on the right hand side of 4.1 is such that some of the factors such as $\Gamma_{\alpha \theta \lambda}$ are equal and interchangeable by the operations of $\Gamma$. Thus if $(\alpha)=\left(\alpha^{\prime}\right)$, $(\theta)=\left(\theta^{\prime}\right)$ so that $\Gamma_{\alpha \theta \lambda}=\Gamma_{\alpha^{\prime} \theta^{\prime} \lambda}$, then in the modified expression the coefficient is taken as $\Gamma_{\alpha \theta \lambda}$ rather than $\Gamma_{\alpha \theta \lambda}^{2}$. The remaining terms are halved in number, 
that is, the coefficient is taken as $\frac{1}{2}\left(\Gamma_{\alpha \theta \lambda}^{2}-\Gamma_{\alpha \theta \lambda}\right)$, and treated as if they belonged to the subgroup which does not interchange $\Gamma_{\alpha \theta \lambda}$ and $\Gamma_{\alpha^{\prime} \theta^{\prime} \lambda}$. In the case when $\Gamma_{\alpha \theta \lambda}, \Gamma_{\alpha^{\prime} \theta^{\prime} \lambda}, \Gamma_{\beta \phi \mu}, \Gamma_{\beta^{\prime} \phi^{\prime} \mu}$ are all identical, say when

$$
(\alpha)=(\theta)=\left(\alpha^{\prime}\right)=\left(\theta^{\prime}\right)=(\beta)=(\phi)=\left(\beta^{\prime}\right)=\left(\phi^{\prime}\right)
$$

the completely modified form is taken with coefficient $\Gamma_{\alpha \theta \lambda}$. Each of 3 subgroups which only interchange the coefficients in pairs is taken with coefficient $\frac{1}{2}\left(\Gamma_{\alpha \theta \lambda}^{2}-\Gamma_{\alpha \theta \lambda}\right)$. Each of two subgroups which make one interchange of coefficients is taken with coefficient $\frac{1}{4}\left(\Gamma_{\alpha \theta \lambda}^{3}-\Gamma_{\alpha \theta \lambda}^{2}\right)$. Finally the completely unmodified term as in (4.1) is taken with coefficient

$$
\frac{1}{8}\left(\Gamma_{\alpha \theta \lambda}^{4}-2 \Gamma_{\alpha \theta \lambda}^{3}-\Gamma_{\alpha \theta \lambda}^{2}+2 \Gamma_{\alpha \theta \lambda}\right) .
$$

The reason for this is that, when $\Gamma_{\alpha \theta \lambda}>1$ the $\Gamma_{\alpha \theta \lambda}$ distinct terms correspond to different Young Tableaux, and interchange is only allowable if the same Young Tableau is involved. For different Young Tableaux, the interchange not being allowable, one half only of the terms are selected.

Although the application of the Theorem for large partitions $(\lambda)$ can be so complicated as to be quite beyond calculation, nevertheless for partitions $(\lambda)$ of $n$ up to, say, $n=5$ application can be quite simple. Thus consider $\left(\{3\} \otimes\left\{1^{2}\right\}\right) \odot\{2\}$.

In every case $(\alpha)=\left(\alpha^{\prime}\right)=(\beta)=\left(\beta^{\prime}\right),(\theta)=\left(\theta^{\prime}\right)=(\phi)=\left(\phi^{\prime}\right)$. In every case $\Gamma=I, S^{2}, T, T S^{2}$ there is the added simplification that in each case

$$
\{\alpha\} \odot\left\{1^{2}\right\}=\{\theta\} \odot\left\{1^{2}\right\}=0 .
$$

Just two cases arise,

$$
(\alpha)=(3),(\theta)=(0) ;(\alpha)=(2),(\theta)=(1)
$$

with $\Gamma_{\alpha \theta \lambda}=1$ in each case.

Hence

$$
\begin{aligned}
\left(\{3\} \otimes\left\{1^{2}\right\}\right) \odot\{2\} & =\{3\} \otimes\{2\}+(\{2\} \otimes\{2\})\{2\} \\
& =2\{6\}+\{51\}+3\{42\}+\{321\}+\left\{2^{3}\right\} .
\end{aligned}
$$

5. Generalizations to Higher Degrees. The above results can be generalized to give expressions for $(\{\lambda\}\{\mu\}) \odot\{\nu\}$ for $(\nu)$ a partition of $n$, and for $(\{\lambda\} \otimes\{\mu\}) \odot\{\nu\}$ for $(\mu)$ a partition of $m,(\nu)$ a partition of $n$. Even in the simplest cases the results alone, without any proofs, are highly elaborate. It may be worth while to indicate briefly the method.

Consider first the case

$$
(\{m\}\{n\}) \odot\{3\}
$$

where $m, n$ are integers. The third induced matrix is considered of the permutation matrix corresponding to sets of $m$ symbols $\alpha_{1}, \alpha_{2}, \ldots, \alpha_{m}$ taken from a set of $m+n$ symbols. For this, sets of 3 rows are considered, possibly with 
repetitions. Suppose that there are $a_{111}$ symbols which are present for all 3 rows, $a_{112}$ symbols which are present for the first two rows but absent from the third, and so on with finally $a_{222}$ symbols absent from the sets for all 3 rows. Then permutation is allowable on each of the respective sets of $a_{111}$, $a_{112}, a_{121}, a_{211}, a_{122}, a_{212}, a_{221}, a_{222}$ symbols.

This leads to

$$
(\{m\}\{n\}) \odot\{3\}=\sum\left(\prod\left\{a_{i j k}\right\}\right)
$$

summed for all solutions of

$$
\sum a_{i j k}=m+n, \sum a_{1 j k}=\sum a_{i 1 k}=\sum a_{i j 1}=m .
$$

This result must be modified if the set of numbers $a_{i j k}$ is unaltered by a group of permutations of the suffixes. In this case products of equal S-functions are replaced by appropriate plethysms.

The modifications for $(\{m\}\{n\}) \odot\{21\}$ and $(\{m\}\{n\}) \odot\left\{1^{3}\right\}$ present no difficulty.

If $\{m\}\{n\}$ is replaced by $\{\mu\}\{\nu\}$, with $(\mu)$ a partition of $m,(\nu)$ a partition of $n$, then $a_{i j k}$ is replaced by a partition $\left(\alpha_{i j k}\right)$.

In the place of equation, say

$$
a_{111}+a_{112}+a_{121}+a_{122}=m,
$$

there is the condition that $\{\mu\}$ appears in the product

$$
\left\{\alpha_{111}\right\}\left\{\alpha_{112}\right\}\left\{\alpha_{121}\right\}\left\{\alpha_{122}\right\}
$$

and $\{\nu\}$ in the product

$$
\left\{\alpha_{211}\right\}\left\{\alpha_{212}\right\}\left\{\alpha_{221}\right\}\left\{\alpha_{222}\right\},
$$

the appropriate coefficients being introduced into the sum.

The generalization of $(\{\lambda\} \otimes\{\mu\}) \odot\{\nu\}$ when $(\mu)$ is a partition of 3 requires an analysis of $(\{\lambda\}\{\lambda\}\{\lambda\}) \odot\{\nu\}$. Repeated application of the result for $(\{\lambda\}\{\mu\}) \odot\{\nu\}$ shows that a similar expansion holds for $\left(\{\lambda\}\left\{\lambda^{\prime}\right\}\left\{\lambda^{\prime \prime}\right\}\right) \odot\{\nu\}$. Putting $(\lambda)=\left(\lambda^{\prime}\right)=\left(\lambda^{\prime \prime}\right)$ the product $\{\lambda\}\{\lambda\}\{\lambda\}$ is expressed in terms of plethysms and symmetrizing operators give $(\{\lambda\} \otimes\{\mu\}) \odot\{\nu\}$. The procedure is in every way similar when $(\mu)$ is a partition of 4 or more.

In the analysis of the last section, when $(\mu),(\nu)$ were partitions of 2 , the group $G$ of order 8 played a leading rôle. This group may be represented as the imprimitive group correspondong to $\{2\} \otimes\{2\}$. When $(\nu)$ is a partition of $m$ and $(\nu)$ a partition of $n$ the group which plays the corresponding role is the imprimitive group corresponding to $\{m\} \otimes\{n\}$. The characters and the subgroups of this group have significant applications. ${ }^{2}$

It may be remarked that while this paper was being written Murnaghan (5) has published a result which is equivalent to Theorem II. He gives no proof.

${ }^{2}$ See (3) for analysis of characters of this group. 


\section{REFERENCES}

1. D. E. Littlewood, The theory of group characters and matrix representations of groups (2nd ed., Oxford, 1950).

2. - The Kronecker product of symmetric group representations, J. London Math. Soc., 31 (1956), 89-93.

3. - The characters and representations of imprimitive groups, Proc. London Math. Soc., (3) 6 (1956), 251-266.

4. - Plethysm and the inner product of S-functions, J. London Math. Soc., 32 (1957), 18-22.

5. F. D. Murnaghan, On the generation of irreducible representations of the symmetric group, Proc. Nat. Acad. Sci., U.S.A., 41 (1955), 514-515.

6. G. de B. Robinson, On the representations of the symmetric group, III. Amer. J. Math. 70 (1948), 277-294.

University College of North Wales, Bangor 